\title{
TRILINEAR ANOMALOUS GAUGE COUPLINGS AND NON-STANDARD FERMIONIC COUPLINGS
}

\author{
R. Casalbuoni, S. De Curtis \\ Dipartimento di Fisica, Univ. di Firenze \\ I.N.F.N., Sezione di Firenze \\ D. Guetta \\ Dipartimento di Fisica, Univ. di Bologna \\ I.N.F.N., Sezione di Bologna
}

University of Florence - DFF 258/10/96 


\begin{abstract}
In this paper we do an analysis of the reaction $e^{+} e^{-} \rightarrow W^{+} W^{-}$for various options of the Next Linear Colliders (NLC), by considering the possibility of deviations from the Standard Model induced by anomalous trilinear vector boson couplings and non-standard fermionic couplings. We show that there are strong correlations among these parameters. By studying the high-energy behaviour of the helicity amplitudes we show that the error made in linearizing the cross-section in the anomalous and non-standard couplings increases with the square of the center of mass energy. We consider also the option of longitudinally polarized electron beams by showing that, in this case, the restrictions on the anomalous and non-standard parameters are greatly enhanced.
\end{abstract}




\section{Introduction}

The sector of the Standard Model (SM) which has been directly verified is the one of the interactions of fermions with gauge vector bosons. However, the non-abelian structure of the SM has been tested only in a very poor manner and indirectly through radiative corrections. The present and future linear collider experiments will offer the opportunity to measure the vector boson self-couplings at a high precision level [四]. This will be done via pair production processes of $W, Z$ and $\gamma$. The first goal of such experiments will be a confirmation of the SM predictions. Besides, they may be used to probe for new physics. In particular it is possible that signals for new physics will appear in the gauge boson sector through the discovery of anomalous vertices.

In this paper we will study the reaction $e^{+} e^{-} \rightarrow W^{+} W^{-}$[2] with the $W$-pair decaying into a lepton pair plus jets. We will consider possible deviations from the SM predictions coming both from anomalous gauge vector boson trilinear couplings and from non-standard couplings of the gauge vector bosons to fermions. Also deviations of the $W$-mass from its SM value will be taken into account. The reason for this study is that we can hardly imagine a scenario of new physics in which only the trilinear couplings are modified in a significant way from their SM values. As an example, let us consider the case of new physics leading to oblique corrections. The modifications induced in the $Z$ propagator will affect both the $Z$-coupling to a $W$-pair and the $Z$-coupling to a fermion pair, and we expect the deviations of the trilinear couplings to be of the same order of magnitude of the deviations in the fermionic couplings. In general the trilinear and the fermionic couplings result strongly correlated (see later), therefore it is not justified to neglect the latter in a phenomenological analysis. Furthermore we expect both these deviations to be small due to the LEP1 bounds on the fermionic couplings. The assumption of smallness of the trilinear anomalous couplings is also compatible with the fact that LEP1 has tested the SM at the level of radiative corrections.

In the present study we will consider also the experimental possibilities of having some polarization [3] in the incoming electron beam and of reconstructing the polarization of the final $W$ 's, because this should increase the sensitivity to the anomalous couplings 叫. Theoretically, this program is carried out by evaluating the helicity amplitudes $F_{\tau \tau^{\prime}}^{\lambda \lambda^{\prime}}$, where $\lambda\left(\lambda^{\prime}\right)$ are the $e^{-}\left(e^{+}\right)$helicities $\left(\lambda^{\prime}=-\lambda= \pm 1 / 2\right)$, and $\tau\left(\tau^{\prime}\right)= \pm 1,0$ are the $W^{-}\left(W^{+}\right)$helicities. In the calculation of the differential cross-sections we have not made any approximation in the anomalous couplings. In fact, as it turns out from the study of the high-energy behaviour, the quadratic terms in these couplings are more and more important as the energy increases.

In Section 2 we will write the general parametrization of the gauge boson trilinear anomalous couplings and of the non-standard fermionic couplings. We will also evaluate the various helicity amplitudes. In Section 3 we will analyze the high-energy behaviour 
of the cross-sections relevant to our study and we will comment about the expansion in the anomalous parameters. In Section 4 we will introduce the observables considered in our analysis and we will discuss the experimental errors. In Section 5 we will show the constraints on the anomalous couplings from future $e^{+} e^{-}$colliders. The options we will consider are for center of mass energies of 360, 500 and $800 \mathrm{GeV}$, with corresponding luminosities of 10, 20 and $50 \mathrm{fb}^{-1}$. Although a general fit could be possible we have limited our analysis to the case of two parameters, by choosing one as an anomalous trilinear coupling, and the other proportional to non-standard fermionic couplings. For completeness we will give in Appendix A the high-energy expansion of the helicity amplitudes.

\section{Helicity Amplitudes}

Let us now start the evaluation of the helicity amplitudes by defining the anomalous couplings we are interested in. We will consider only the $C$ and $P$ invariant possible trilinear couplings as defined by the lagrangian [5]

$$
\begin{aligned}
\mathcal{L}_{\text {eff }}^{3}= & -i e\left[A_{\mu}\left(W^{-\mu \nu} W_{\nu}^{+}-W^{+\mu \nu} W_{\nu}^{-}\right)+A^{\mu \nu} W_{\mu}^{+} W_{\nu}^{-}\right]-i e x_{\gamma} A^{\mu \nu} W_{\mu}^{+} W_{\nu}^{-} \\
& -i e\left(\operatorname{ctg} \theta+\delta_{z}\right)\left[Z_{\mu}\left(W^{-\mu \nu} W_{\nu}^{+}-W^{+\mu \nu} W_{\nu}^{-}\right)\right]-i e x_{z} Z^{\mu \nu} W_{\mu}^{+} W_{\nu}^{-} \\
& +i e \frac{y_{\gamma}}{M_{W}^{2}} A^{\nu \lambda} W_{\lambda \mu}^{-} W_{\nu}^{+\mu}+i e \frac{y_{z}}{M_{W}^{2}} Z^{\nu \lambda} W_{\lambda \mu}^{-} W_{\nu}^{+\mu}
\end{aligned}
$$

where $V_{\mu \nu}=\partial_{\mu} V_{\nu}-\partial_{\nu} V_{\mu}$ for $V=A, Z, W$. As it is known, the parameters $x_{\gamma}$ and $x_{z}$ are related to the deviations of the dipole couplings from their SM values. The parameters $y_{\gamma}$ and $y_{z}$ give non-zero quadrupole interactions and $\delta_{z}$ is an overall deviation of the SM $Z W^{+} W^{-}$coupling.

We will assume that the energy relevant to the problem (i.e. the energy of the electronpositron beams) is much lower than the energy scale of the new physics. As a consequence we will work in the spirit of the effective lagrangians, by assuming all the anomalous couplings as constant and ignoring their possible energy dependence.

The relevant charged and neutral couplings of $W$ and $Z$ to fermions are defined by the following lagrangians

$$
\begin{gathered}
\mathcal{L}^{\text {neutral }}=-e Z_{\mu} \bar{\psi}_{e}\left[\gamma^{\mu} a_{e}^{Z}-\gamma^{\mu} \gamma_{5} b_{e}^{Z}\right] \psi_{e}-e A_{\mu} \bar{\psi}_{e} Q_{e} \psi_{e} \\
\mathcal{L}^{\text {charged }}=e a_{w}\left(\sum_{\ell} \bar{\psi}_{\nu_{\ell}} \gamma^{\mu}\left(1-\gamma_{5}\right) \psi_{\ell} W_{\mu}^{+}+\sum_{i=1}^{3} \bar{\psi}_{i} \gamma^{\mu}\left(1-\gamma_{5}\right) \tau_{+} \psi_{i} W_{\mu}^{+}+\text {h.c. }\right)
\end{gathered}
$$

In these expressions $\psi_{e}$ is the electron field, $\ell=e, \mu, \tau$, and the sum over $i=1,2,3$ is on the quark charged current eigenstates. In the previous equations we will put the 
parameters $a_{e}^{Z}, b_{e}^{Z}$ and $a_{W}$ equal to their SM values plus anomalous terms, i.e.

$$
\begin{aligned}
a_{e}^{Z} & =a_{e}^{Z}(S M)+\delta a_{e}^{Z} \\
b_{e}^{Z} & =b_{e}^{Z}(S M)+\delta b_{e}^{Z} \\
a_{W} & =a_{W}(S M)+\delta a_{W}
\end{aligned}
$$

with

$$
\begin{gathered}
a_{e}^{Z}(S M)=\frac{1}{2 s_{\theta} c_{\theta}}\left(-\frac{1}{2}+2 s_{\theta}^{2}\right) \\
b_{e}^{Z}(S M)=-\frac{1}{4 s_{\theta} c_{\theta}} \\
a_{W}(S M)=\frac{1}{2 \sqrt{2} s_{\theta}}
\end{gathered}
$$

where $s_{\theta}$ is defined through the input parameters $G_{F}, \alpha\left(M_{Z}\right), M_{Z}$

$$
\frac{G_{F}}{\sqrt{2}}=\frac{4 \pi \alpha\left(M_{Z}\right)}{8 s_{\theta}^{2} c_{\theta}^{2} M_{Z}^{2}}
$$

or

$$
s_{\theta}^{2}=\frac{1}{2}-\sqrt{\frac{1}{4}-\frac{\pi \alpha\left(M_{Z}\right)}{\sqrt{2} G_{F} M_{Z}^{2}}}
$$

Finally we put

$$
M_{W}=M_{W}(S M)+\delta M_{W}
$$

with

$$
M_{W}(S M)=c_{\theta} M_{Z}
$$

The differential cross-section for initial $e_{\lambda^{\prime}}^{+}, e_{\lambda}^{-}$, and final $W_{\tau^{\prime}}^{+}, W_{\tau}^{-}$is given by

$$
\frac{d \sigma_{\tau \tau^{\prime}}^{\lambda \lambda^{\prime}}}{d \cos \phi}=\frac{|\vec{p}|}{4 \pi s \sqrt{s}}\left|F_{\tau \tau^{\prime}}^{\lambda \lambda^{\prime}}(s, \cos \phi)\right|^{2}
$$

where

$$
|\vec{p}|=\beta_{W} \frac{\sqrt{s}}{2}, \quad \beta_{W}=\sqrt{1-\frac{4 M_{W}^{2}}{s}}
$$

and $\phi$ is the angle between the incoming electron and $W^{-}$.

To express the results for the helicity amplitudes we will use the same formulation as given in ref. [6]. We start separating the contributions from photon, $Z$, and neutrino

$$
F_{\tau \tau^{\prime}}^{\lambda \lambda^{\prime}}=F=F_{\gamma}+F_{Z}+F_{\nu}
$$


Then we define reduced amplitudes $\tilde{F}$ by factorizing out the leading angular dependence in terms of the rotation functions $d_{\mu, \mu^{\prime}}^{J}$. Here $J=1,2$ corresponds to the lowest angular momentum which contributes to a given helicity amplitude. We will write

$$
F_{\tau \tau^{\prime}}^{\lambda \lambda \lambda^{\prime}}=\sqrt{2} \lambda e^{2} d_{2 \lambda, \tau-\tau^{\prime}}^{J} \tilde{F}_{\tau \tau^{\prime}}^{\lambda \lambda^{\prime}}
$$

We follow here the phase conventions as in Gounaris et al. in ref. [6]. However, our helicity amplitudes $F$ correspond to their $\mathcal{M} / 2$. In the case of transverse final $W$ 's, and for $\left(\tau, \tau^{\prime}\right)=( \pm 1, \mp 1)$, corresponding to $J=2$, the electron must be left-handed, and the helicity amplitude takes contribution only from the neutrino exchange. We obtain

$$
F_{\tau,-\tau}^{-1 / 2,1 / 2}=-4 \tau e^{2}\left(\frac{1}{2 \sqrt{2} s_{\theta}}+\delta a_{W}\right)^{2} \frac{s}{t} \sin \phi\left(\frac{1-\tau \cos \phi}{2}\right)
$$

The other helicity amplitudes involve all the contributions and their reduced amplitudes can be expressed in the following way (separating the various contributions)

$$
\begin{gathered}
\tilde{F}_{\gamma}=-\beta_{W} A^{\gamma} \\
\tilde{F}_{Z}=\beta_{W} A^{Z}\left(\delta_{\lambda, 1 / 2}\left(1+\cot \theta\left(\delta a_{e}^{Z}-\delta b_{e}^{Z}\right)\right)\right. \\
\left.+\delta_{\lambda,-1 / 2}\left(1-\frac{1}{2 s_{\theta}^{2}}+\cot \theta\left(\delta a_{e}^{Z}+\delta b_{e}^{Z}\right)\right)\right) \frac{s}{s-M_{Z}^{2}} \\
\tilde{F}_{\nu}=\delta_{\lambda,-1 / 2} \frac{4}{\beta_{W}}\left(\frac{1}{2 \sqrt{2} s_{\theta}}+\delta a_{W}\right)^{2}\left(B_{\tau \tau^{\prime}}+\frac{s}{4 t} C_{\tau \tau^{\prime}}\right)
\end{gathered}
$$

where the Mandelstam variable $t$ is defined by

$$
t=M_{W}^{2}-\frac{s}{2}\left(1-\beta_{W} \cos \phi\right)
$$

and the coefficients $A^{V}, B$ and $C$ are given in Table 1 . Here, $V=\gamma, Z$, and we have used the following definitions

$$
\begin{gathered}
g_{1}^{\gamma}=1, \quad g_{1}^{Z}=1+\delta_{z} \tan \theta \\
\kappa_{\gamma}=1+x_{\gamma}, \quad \kappa_{Z}=1+\left(x_{z}+\delta_{z}\right) \tan \theta \\
\lambda_{\gamma}=y_{\gamma}, \quad \lambda_{Z}=y_{z} \tan \theta
\end{gathered}
$$

It is worth to notice that the helicity amplitudes for two longitudinally polarized $W$ 's do not depend on the quadrupole couplings $y_{z}$ and $y_{\gamma}$. 
Table 1: Reduced helicity amplitudes $\tilde{F}_{\tau \tau^{\prime}}^{\lambda \lambda \prime^{\prime}}$, for the process $e^{+} e^{-} \rightarrow W^{+} W^{-}$.

\begin{tabular}{|c||c|c|c|c|}
\hline$\left(\tau, \tau^{\prime}\right)$ & $A_{\tau, \tau^{\prime}}^{V}$ & $B_{\tau, \tau^{\prime}}$ & $C_{\tau, \tau^{\prime}}$ & $d_{2 \lambda, \tau-\tau^{\prime}}^{J}$ \\
\hline$(+1,+1)$ & $g_{1}^{V}+\frac{s}{2 M_{W}^{2}} \lambda_{V}$ & 1 & $\frac{4 M_{W}^{2}}{s}$ & $-\frac{2 \lambda}{\sqrt{2}} \sin \phi$ \\
\hline$(-1,-1)$ & $g_{1}^{V}+\frac{s}{2 M_{W}^{2}} \lambda_{V}$ & 1 & $\frac{4 M_{W}^{2}}{s}$ & $-\frac{2 \lambda}{\sqrt{2}} \sin \phi$ \\
\hline$(+1,0)$ & $\frac{\sqrt{s}}{2 M_{W}}\left(g_{1}^{V}+\kappa_{V}+\lambda_{V}\right)$ & $\frac{\sqrt{s}}{M_{W}}$ & $\frac{4 M_{W}}{\sqrt{s}}\left(1+\beta_{W}\right)$ & $\frac{(1+2 \lambda \cos \phi)}{2}$ \\
\hline$(0,-1)$ & $\frac{\sqrt{s}}{2 M_{W}}\left(g_{1}^{V}+\kappa_{V}+\lambda_{V}\right)$ & $\frac{\sqrt{s}}{M_{W}}$ & $\frac{4 M_{W}}{\sqrt{s}}\left(1+\beta_{W}\right)$ & $\frac{(1+2 \lambda \cos \phi)}{2}$ \\
\hline$(0,+1)$ & $\frac{\sqrt{s}}{2 M_{W}}\left(g_{1}^{V}+\kappa_{V}+\lambda_{V}\right)$ & $\frac{\sqrt{s}}{M_{W}}$ & $\frac{4 M_{W}}{\sqrt{s}}\left(1-\beta_{W}\right)$ & $\frac{(1-2 \lambda \cos \phi)}{2}$ \\
\hline$(-1,0)$ & $\frac{\sqrt{s}}{2 M_{W}}\left(g_{1}^{V}+\kappa_{V}+\lambda_{V}\right)$ & $\frac{\sqrt{s}}{M_{W}}$ & $\frac{4 M_{W}}{\sqrt{s}}\left(1-\beta_{W}\right)$ & $\frac{(1-2 \lambda \cos \phi)}{2}$ \\
\hline$g_{1}^{V}+\frac{s}{2 M_{W}^{2}} \kappa_{V}$ & $\frac{s}{2 M_{W}^{2}}$ & $\frac{8 M_{W}^{2}}{s}$ & $-\frac{2 \lambda}{\sqrt{2}} \sin \phi$ \\
\hline
\end{tabular}




\section{High-energy behaviour}

In order to have a qualitative idea of the behaviour of the helicity amplitudes, we will consider here a double expansion in powers of the energy and of the anomalous couplings. In the phenomenological analysis, however, we will use the exact expressions.

Within the SM the $e^{+} e^{-} \rightarrow W^{+} W^{-}$cross-section has a good behaviour at highenergy due to the cancellations required by the gauge-invariance. In the actual case, however, this is not so. We should remember that we are dealing with an effective theory and that we are allowed to use it only up to energies smaller with respect to the new physics scale. The analysis of the high-energy behaviour is particularly simple in terms of the helicity amplitudes, in fact the cancellations operating at the level of the SM must be effective within each single helicity amplitude (remember that the cross-section is a sum of modulus squares of helicity amplitudes). The leading behaviour in energy is simply evaluated just counting the powers of energy associated to the various vertices, propagators and external wave functions. The worst behaved amplitudes are the ones corresponding to the production of two longitudinal vector bosons. The power counting would imply that the corresponding amplitudes (let us call them shortly $F_{L L}$ ) should increase as $s^{2}$, due to the fact that the vertices coming from the quadrupole interactions contain three derivatives. This is not the case since, as already noticed, these amplitudes do not depend on the couplings $y_{z}$ and $y_{\gamma}$ signalling that the quadrupole vertices do not contribute to $F_{L L}$. In fact we can show that, due to the structure of the quadrupole interaction, the corresponding high-energy behaviour of the helicity amplitudes is not the one coming from simple power counting but it is suppressed by one power of $s$. The quadrupole interactions are made up entirely in terms of the abelian field strengths $A_{\mu \nu}$, $Z_{\mu \nu}$ and $W_{\mu \nu}$, therefore if we decompose the helicity amplitudes in the form

$$
F_{\tau \tau^{\prime}}^{\lambda \lambda^{\prime}}=F_{\mu \mu^{\prime}}^{\lambda \lambda^{\prime}} \epsilon_{\tau}^{\mu}\left(p^{-}\right) \epsilon_{\tau^{\prime}}^{\mu^{\prime}}\left(p^{+}\right)
$$

where $p^{ \pm}$are the momenta of $W^{ \pm}$and $\epsilon_{\tau}^{\mu}$ is the polarization vector for the $W^{\prime}$ s, the quadrupole parts of the amplitudes must satisfy

$$
\left.\left.\left(p^{-}\right)^{\mu} F_{\mu \mu^{\prime}}^{\lambda \lambda^{\prime}} \text { (quadr. }\right)=\left(p^{+}\right)^{\mu^{\prime}} F_{\mu \mu^{\prime}}^{\lambda \lambda^{\prime}} \text { (quadr. }\right)=0
$$

In the high-energy limit the longitudinal polarization vectors have the following behaviour

$$
\epsilon_{L}^{\mu}(p)=\frac{p^{\mu}}{M_{W}}\left(1+\mathcal{O}\left(\frac{1}{s}\right)\right)
$$

It follows that $y_{z}$ and $y_{\gamma}$ cannot give contribution to the highest possible power in $s$ in amplitudes relative to longitudinally polarized $W^{\prime}$ 's. As a consequence the highest power for $F_{L L}$ is $s$ instead of $s^{2}$, whereas for $F_{T L}$ it is $s^{1 / 2}$ instead of $s^{3 / 2}$. 
Here we will consider the corrections to the helicity amplitudes up to the first order in the anomalous couplings and in $\delta M_{W}$ (see eq. (2.10))

$$
F_{\tau \tau^{\prime}}^{\lambda \lambda^{\prime}}=F_{\tau \tau^{\prime}}^{\lambda \lambda^{\prime}}(S M)+\delta F_{\tau \tau^{\prime}}^{\lambda \lambda^{\prime}}
$$

where $F_{\tau \tau^{\prime}}^{\lambda \lambda \gamma^{\prime}}(S M)$ are the SM helicity amplitudes, and then we will expand the generic helicity amplitude, $F$, in powers of energy

$$
F=\sum_{\alpha} F^{(\alpha)} s^{\alpha}
$$

One gets, for the longitudinal-longitudinal case

$$
F_{L L}=\delta F_{L L}^{(1)} s+F_{L L}^{(0)}(S M)+\delta F_{L L}^{(0)}+\frac{1}{s}\left(F_{L L}^{(-1)}(S M)+\delta F_{L L}^{(-1)}\right)+\mathcal{O}\left(\frac{1}{s^{2}}\right)
$$

for the longitudinal-transverse case

$$
F_{L T}=\sqrt{s}\left(\delta F_{L T}^{(1 / 2)}+\frac{1}{s}\left(F_{L T}^{(-1 / 2)}(S M)+\delta F_{L T}^{(-1 / 2)}\right)+\mathcal{O}\left(\frac{1}{s^{2}}\right)\right)
$$

and finally the expansion for $F_{T T}$ is

$$
F_{T T}=\delta F_{T T}^{(1)} s+F_{T T}^{(0)}(S M)+\delta F_{T T}^{(0)}+\frac{1}{s}\left(F_{T T}^{(-1)}(S M)+\delta F_{T T}^{(-1)}\right)+\mathcal{O}\left(\frac{1}{s^{2}}\right)
$$

Notice that $F_{T T}$ has only contributions from $y_{z}$ and $y_{\gamma}$, because in this case they are not suppressed and, by power counting, the other anomalous contributions should give a constant term for $s \rightarrow \infty$. The list of the coefficients $F^{(\alpha)}$ is given in Appendix A.

We can now discuss the anomalous contributions in the energy expansion of the differential cross-sections. We get (we do not expand the threshold factor $\beta_{W}$, see eqs. (2.12) and (2.13));

$$
\begin{aligned}
\frac{d \sigma_{L L}}{d \cos \phi} & =\frac{\beta_{W}}{8 \pi}\left[\left|\delta F_{L L}^{(1)}\right|^{2} s+\left(\delta F_{L L}^{(1)}\right)^{*} F_{L L}^{(0)}(S M)+\delta F_{L L}^{(1)}\left(F_{L L}^{(0)}(S M)\right)^{*}\right. \\
& \left.+\frac{1}{s} \sum_{\alpha=1,0}\left(\delta F_{L L}^{(\alpha)}\right)^{*} F_{L L}^{(-\alpha)}(S M)+\delta F_{L L}^{(\alpha)}\left(F_{L L}^{(-\alpha)}(S M)\right)^{*}\right]+\mathcal{O}\left(\frac{1}{s^{2}}\right) \\
\frac{d \sigma_{L T}}{d \cos \phi}= & \frac{\beta_{W}}{8 \pi}\left[\left|\delta F_{L T}^{(1)}\right|^{2}+\frac{1}{s}\left(\left(\delta F_{L T}^{(1 / 2)}\right)^{*} F_{L T}^{(-1 / 2)}(S M)+\delta F_{L T}^{(1 / 2)}\left(F_{L T}^{(-1 / 2)}(S M)\right)^{*}\right)\right] \\
& +\mathcal{O}\left(\frac{1}{s^{2}}\right)
\end{aligned}
$$




$$
\begin{aligned}
\frac{d \sigma_{T T}}{d \cos \phi} & =\frac{\beta_{W}}{8 \pi}\left[\left|\delta F_{T T}^{(1)}\right|^{2} s+\left(\delta F_{T T}^{(1)}\right)^{*} F_{T T}^{(0)}(S M)+\delta F_{T T}^{(1)}\left(F_{T T}^{(0)}(S M)\right)^{*}\right. \\
& \left.+\frac{1}{s} \sum_{\alpha=1,0}\left(\delta F_{T T}^{(\alpha)}\right)^{*} F_{T T}^{(-\alpha)}(S M)+\delta F_{T T}^{(\alpha)}\left(F_{T T}^{(-\alpha)}(S M)\right)^{*}\right]+\mathcal{O}\left(\frac{1}{s^{2}}\right)
\end{aligned}
$$

At each order in $s$ we have taken only the first non-zero terms in the anomalous couplings. In particular the terms linear in $s$ turn out to be quadratic in the anomalous couplings. Notice that the true expansion parameter is $s / M_{W}^{2}$, therefore it is a bad approximation to neglect the quadratic terms in the anomalous parameters when one is discussing the cross-sections at energies of the order $10 M_{W}$ or more (we are assuming a size for the anomalous parameters comparable to the size of the radiative corrections within the SM).

As we have already observed $d \sigma_{L L}$ does not depend on $y_{z}$ and $y_{\gamma}$. Furthermore, by looking at the explicit expressions in Appendix A, we see that the most divergent terms in $d \sigma_{L T}$ depend on $x_{\gamma}+y_{\gamma}, 2 \delta_{z}+x_{z}+y_{z}, \delta a_{e}^{Z}, \delta b_{e}^{Z}, \delta a_{W}$ and, in $d \sigma_{T T}$ depend only on $y_{\gamma}, y_{z}$. It should also be noted that $\delta M_{W}$ appears only at lower orders in the expansion. This study suggests that an analysis of the differential cross-sections at various energies should be useful in order to discriminate among various anomalous parameters.

\section{Phenomenological analysis}

In the following phenomenological analysis we will consider differential cross-sections for longitudinally polarized electron and unpolarized positron beams

$$
\frac{d \sigma_{\tau \tau^{\prime}}}{d \cos \phi}=\frac{1}{4}\left[\left(1+P_{e}\right) \frac{d \sigma_{\tau \tau^{\prime}}^{(+1 / 2,-1 / 2)}}{d \cos \phi}+\left(1-P_{e}\right) \frac{d \sigma_{\tau \tau^{\prime}}^{(-1 / 2,+1 / 2)}}{d \cos \phi}\right]
$$

where $P_{e}$ is the degree of longitudinal polarization. For $P_{e}=0$ we get the expression for unpolarized electrons. We have also considered the production of pairs of polarized $W$ 's.

In order to study the sensitivity of the differential cross-sections to the anomalous gauge couplings and to the non-standard fermionic couplings, we divide the experimentally significant range of $\cos \phi(-0.95 \leq \cos \phi \leq 0.95)$ into 6 bins. For each differential crosssection we can evaluate the differences

$$
\sigma_{i, a}^{A N}=\sigma_{i, a}-\sigma_{i, a}(S M), \quad i=1, \cdots, 6, \quad a=L L, L T, T T
$$

where $\sigma_{i, a}$ and $\sigma_{i, a}(S M)$ are respectively the full and the SM differential cross-sections integrated over the bin $i$. From these differences we can evaluate the $\chi^{2}$ function for each differential cross-section

$$
\chi_{a}^{2}=\sum_{i=1}^{6} \frac{\left(\sigma_{i, a}^{A N}\right)^{2}}{\left(\delta \sigma_{i, a}\right)^{2}}, \quad a=L L, L T, T T
$$


where we assume the errors $\delta \sigma_{i, a}$ as follows

$$
\delta \sigma_{i, a}=\sqrt{\frac{\sigma_{i, a}}{B R \cdot L}+\sigma_{i, a}^{2} \Delta_{\mathrm{sys}}^{2}}
$$

where $\Delta_{\text {sys }}$ is the systematic error taking into account the relative errors on the luminosity, on the branching ratio of the decay of $W^{\prime}$ 's into a lepton pair plus jets, on the acceptance and on the longitudinal polarization of the electron beam. We assume $\Delta_{\text {sys }}=1.5 \%$. We take into account the efficiency in reconstructing the $W$ pairs from their decays into a lepton pair plus jets by reducing the true branching ratio from 0.29 to 0.10 (see [7]). This is the effective branching ratio $B R$ that we use in eq. (4.4). There is a delicate point about the branching ratio. In fact we are assuming that the coupling $W e \nu$ may differ from its SM value. This could modify the branching ratios of $W$ 's into fermions. However, for deviation respecting the universality, it is easy to see that neglecting, as usual, mixed terms in the radiative and in the anomalous corrections, the branching ratios are the same as in the SM up to 1-loop level.

\section{Bounds on the anomalous couplings}

In this Section we will consider the bounds on the anomalous couplings coming from the minimization of the $\chi^{2}$-function as defined in the previous Section. We will not attempt to make a general analysis in all the parameters expressing the deviations from the SM ( 5 anomalous trilinear couplings, 3 non-standard fermionic couplings and the $M_{W}$ deviation), but rather we will concentrate on a few models depending only on 2 parameters. The first simplifying hypothesis we will make is that all the fermionic deviations can be expressed in terms of the $\epsilon_{i}, i=1,2,3$, parameters [8]. By using the definitions of the $\epsilon_{i}$ in terms of the observables one gets

$$
\begin{aligned}
\delta a_{e}^{Z} & =-\frac{\epsilon_{1}}{8 s_{\theta} c_{\theta}}-\frac{1}{c_{2 \theta}} \frac{s_{\theta}}{c_{\theta}}\left(\frac{\epsilon_{1}}{2}-\epsilon_{3}\right) \\
\delta b_{e}^{Z} & =\frac{\epsilon_{1}}{8 s_{\theta} c_{\theta}} \\
\delta a_{W} & =\frac{1}{2 \sqrt{2} s_{\theta}}\left(\frac{c_{\theta}^{2}}{2 c_{2 \theta}} \epsilon_{1}-\frac{\epsilon_{2}}{2}-\frac{s_{\theta}^{2}}{c_{2 \theta}} \epsilon_{3}\right) \\
\frac{\delta M_{W}^{2}}{M_{W}^{2}} & =\frac{c_{\theta}^{2}}{c_{2 \theta}} \epsilon_{1}-\epsilon_{2}-2 \frac{s_{\theta}^{2}}{c_{2 \theta}} \epsilon_{3}
\end{aligned}
$$

In many models of new physics there is no isospin violation. In such a case one has $\epsilon_{1}=\epsilon_{2}=0$. We will restrict our analysis to these models, therefore all the non-standard fermionic couplings and $\delta M_{W}$ are parametrized in terms of $\epsilon_{3}$. The experimental bound on $\epsilon_{3}$ coming from LEP1 measurements ref. [9], is

$$
\epsilon_{3}^{\exp }=(4.6 \pm 1.5) \times 10^{-3}
$$


Notice that the $\epsilon_{3}$ appearing in our equations is only the contribution due to new physics, so in order to get an useful bound for it one has to subtract from $\epsilon_{3}^{\exp }$ the contribution from the SM radiative corrections. We will assume in our analysis $m_{\text {top }}=175 \mathrm{GeV}$ and a value of $m_{\text {Higgs }}$ ranging from $65 \mathrm{GeV}$ to $1 \mathrm{TeV}$. The corresponding values due to radiative corrections are

$$
\begin{array}{lll}
\epsilon_{3}^{\mathrm{rad}}=4.65 \times 10^{-3}, & m_{\text {Higgs }}=65 \mathrm{GeV} \\
\epsilon_{3}^{\mathrm{rad}}=6.53 \times 10^{-3}, & m_{\text {Higgs }}=1 \mathrm{TeV}
\end{array}
$$

The $90 \%$ C.L. bound we get (in the case of two degrees of freedom) is then

$$
-\left(4.5_{+0.6}^{-1.3}\right) \times 10^{-3} \leq \epsilon_{3} \leq\left(1.9_{-0.6}^{+1.3}\right) \times 10^{-3}
$$

The upper and lower bounds correspond to $m_{\text {Higgs }}=65 \mathrm{GeV}$ and $m_{\text {Higgs }}=1 \mathrm{TeV}$ respectively. The central value is obtained for $m_{\text {Higgs }}=300 \mathrm{GeV}$, with a corresponding radiative contribution given by

$$
\epsilon_{3}^{\mathrm{rad}}=5.91 \times 10^{-3}, \quad m_{\text {Higgs }}=300 \mathrm{GeV}
$$

A comparable bound on $\epsilon_{3}$ will be obtained by the precision measure of $M_{W}$ at LEP2. Assuming $\delta M_{W}=50 \mathrm{MeV}$ we get from eq. (5.1), always in the hypothesis of $\epsilon_{1}=\epsilon_{2}=0$,

$$
-3.2 \times 10^{-3} \leq \epsilon_{3} \leq 3.2 \times 10^{-3}
$$

As far as the anomalous trilinear gauge couplings are concerned we will also consider some simplified models.

\section{$\underline{\text { MODEL } 1}$}

A first possibility discussed in ref. [10] is to assume a global $S U(2)_{L}$ symmetry for the lagrangian (2.1). This gives rise to the following relations among the trilinear anomalous couplings:

$$
\delta_{z}=x_{\gamma}=x_{z}=0
$$

and

$$
y_{z}=\frac{c_{\theta}}{s_{\theta}} y_{\gamma} \neq 0
$$

In this way, model 1 depends only on the two parameters $\left(\epsilon_{3}, y_{\gamma}\right)$. We analyze the model for various observables, namely the unpolarized cross-sections for different final $W$ 's polarization, $\sigma_{L L}, \sigma_{L T}, \sigma_{T T}$ and the total one. The $90 \%$ C.L. bounds are given in Fig. 1, together with the bounds coming from combining the $\chi^{2}$ for the final state polarized observables, $\sigma_{\text {comb }}$. In the figure we give the bounds for a NLC machine with a center of mass 


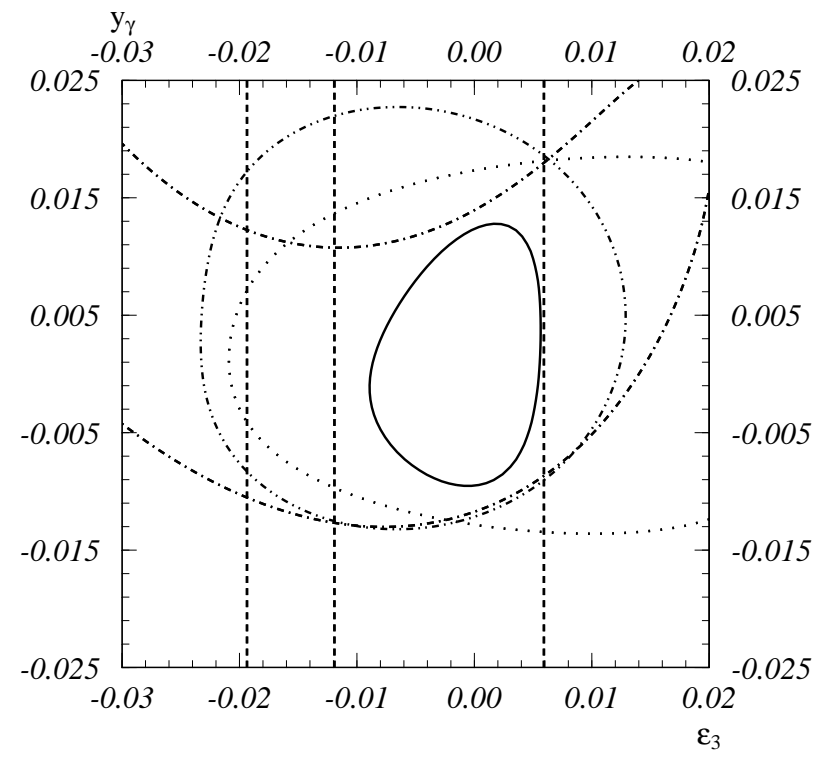

Figure 1: $90 \%$ C.L. bounds on the parameter space $\left(\epsilon_{3}, y_{\gamma}\right)$ in the case of model 1 . The dotted line represents the bounds from $\sigma_{T T}$, the dash-dotted one from $\sigma_{L T}$, the dashed one from $\sigma_{L L}$, the dash-doubledotted one from $\sigma_{\text {total }}$, and the continuous one from $\sigma_{\text {comb }}$. Here the parameters of the NLC are $E=500 \mathrm{GeV}, L=20 \mathrm{fb}^{-1}$, and $P_{e}=0$.

energy of $500 \mathrm{GeV}$ and a luminosity of $20 \mathrm{fb}^{-1}$. The figure shows what we have already discussed before, that is the independence of $\sigma_{L L}$ on $y_{\gamma}$. In this case it is important to measure other differential cross-sections. Also the total one, if combined with $\sigma_{L L}$ would give a good restriction in the parameter space. In Fig. 2 we give the $90 \%$ C.L. bounds arising from combining the final state polarized differential cross-sections at various energies and luminosities, namely: $E=360 \mathrm{GeV}, L=10 \mathrm{fb}^{-1} ; E=500 \mathrm{GeV}, L=20 \mathrm{fb}^{-1}$; $E=800 \mathrm{GeV}, L=50 \mathrm{fb}^{-1}$. We see that one needs to reach at least $800 \mathrm{GeV}$ in energy in order to get bounds on $\epsilon_{3}$ comparable with the ones obtained at LEP1 also shown in the figure. The corresponding strip is for $65 \leq m_{H}(\mathrm{GeV}) \leq 1000$. Notice that the bound at $E=800 \mathrm{GeV}$ includes also a region which is not connected with the SM point. This means that there is the possibility of having an ambiguity at this energy. However this is already resolved by the LEP1 data. It is also interesting to notice that there are strong correlations among $y_{\gamma}$ and $\epsilon_{3}$. This means that going at values of $\epsilon_{3}$ different from zero, the bounds on $y_{\gamma}$ can change considerably. As we will see this is a rather general feature.

\section{MODEL 2}

Next we consider the case in which one adds to the SM lagrangian the most general operator of dimension 6 invariant under $S U(2)_{L} \otimes U(1)$ (see ref. [10]). The general form 


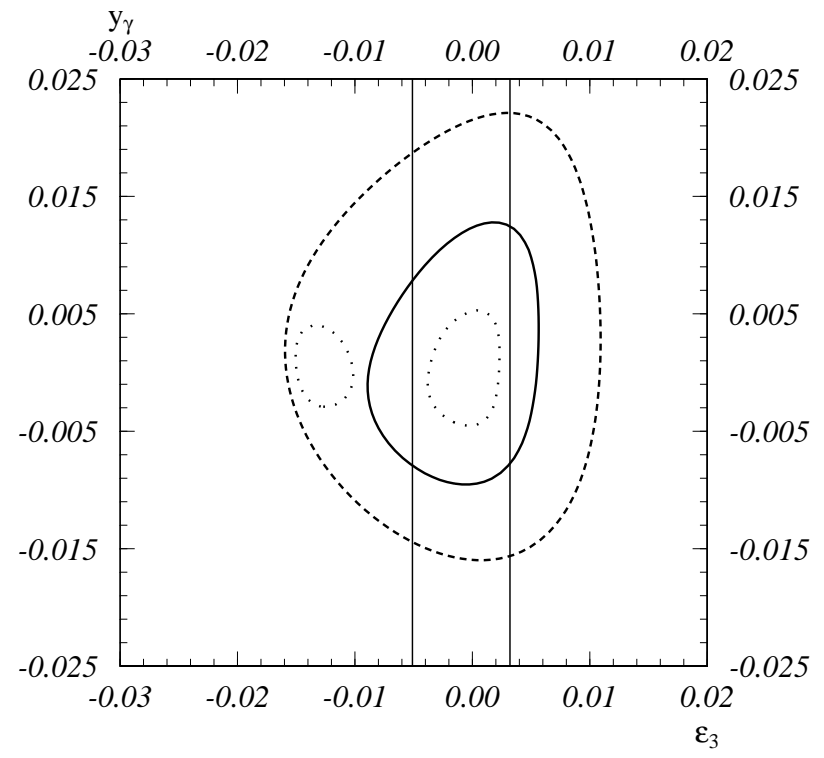

Figure 2: $90 \%$ C.L. bounds on the parameter space $\left(\epsilon_{3}, y_{\gamma}\right)$ in the case of model 1 . The bounds are obtained from $\sigma_{\text {comb }}$ at various energies and luminosities. The dashed line corresponds to $E=360 \mathrm{GeV}$ and $L=10 \mathrm{fb}^{-1}$, the continuous one to $E=500 \mathrm{GeV}$ and $L=20 \mathrm{fb}^{-1}$, and the dotted one to $E=800 \mathrm{GeV}, L=50 \mathrm{fb}^{-1}$, and $P_{e}=0$ The vertical lines correspond to the LEP1 data.

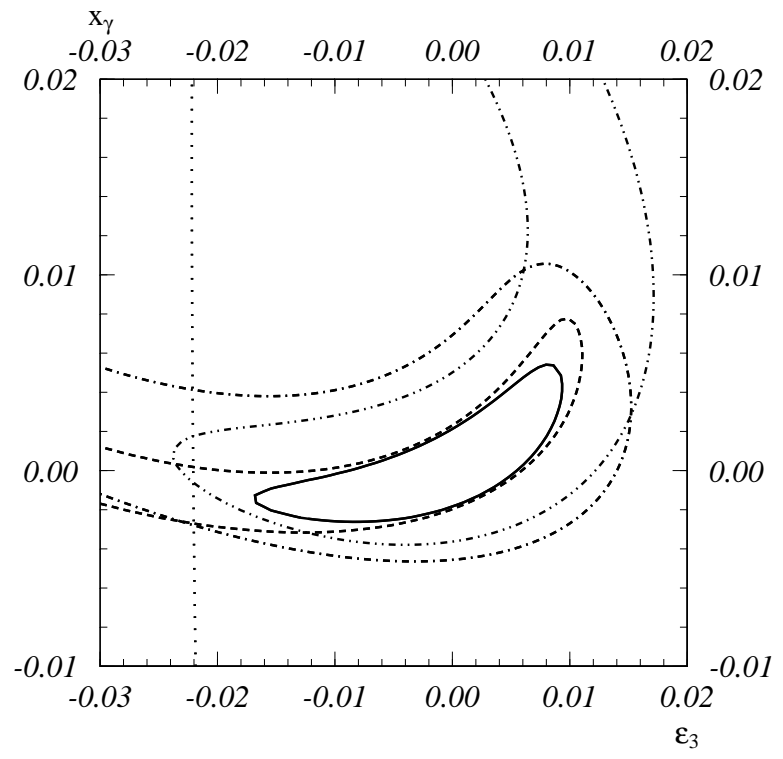

Figure 3: Same of Figure 1 for the parameter space $\left(\epsilon_{3}, x_{\gamma}\right)$ in the case of model 2. 


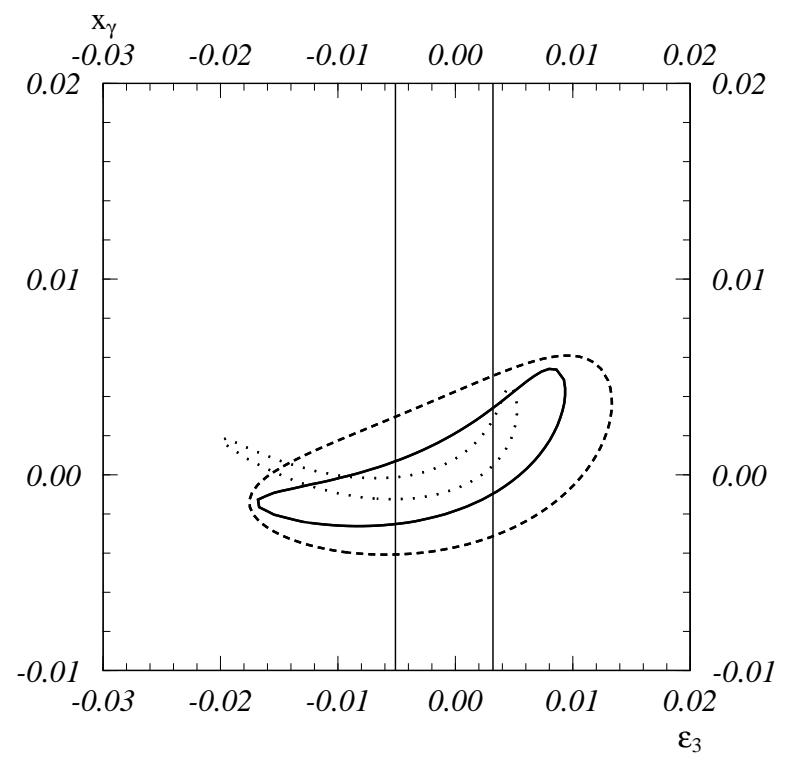

Figure 4: Same of Figure 2 for the parameter space $\left(\epsilon_{3}, x_{\gamma}\right)$ in the case of model 2.

of this term is

$$
\frac{i e}{2 M_{W}^{2}}\left[\frac{f_{B}}{c_{\theta}} B_{\mu \nu}\left(D_{\mu} \phi\right)^{\dagger}\left(D_{\nu} \phi\right)+\frac{f_{w}}{s_{\theta}} \vec{w}_{\mu \nu} \cdot\left(D_{\mu} \phi\right)^{\dagger} \vec{\tau}\left(D_{\nu} \phi\right)\right]
$$

where $B_{\mu \nu}$ is the weak hypercharge field strength, $\vec{w}_{\mu \nu}$ is the non-abelian field strength associated to $\vec{W}_{\mu}$ and $D_{\mu}$ the covariant derivative operating on the Higgs field $\phi$. The interaction generated by this operator is non-renormalizable but, assuming $f_{B}=f_{w}$, the divergences soften and only the logarithmic ones remain in the final result, whereas the potential quadratic divergences are replaced by quadratic dependence on the Higgs mass. The operator (5.9) induces anomalous couplings which, with the further constraint $f_{B}=f_{w}$, satisfy the following relations

$$
x_{z}=-\frac{s_{\theta}}{c_{\theta}} x_{\gamma}, \quad \delta_{z}=\frac{2}{s_{\theta} c_{\theta}} x_{\gamma}, \quad y_{\gamma}=y_{z}=0
$$

Assuming again $\epsilon_{3} \neq 0$ we get a two parameter space $\left(\epsilon_{3}, x_{\gamma}\right)$. Fig. 3 is the analogous of Fig. 1 for the present model. We notice that $\sigma_{L L}$ bounds strongly $x_{\gamma}$ but has a weaker influence on $\epsilon_{3}$. Also $\sigma_{T T}$ does not depend on $x_{\gamma}$. In Fig. 4 we give again the bounds for various energies and luminosities. Finally let us stress once more the strong correlation among the trilinear anomalous coupling and $\epsilon_{3}$. 


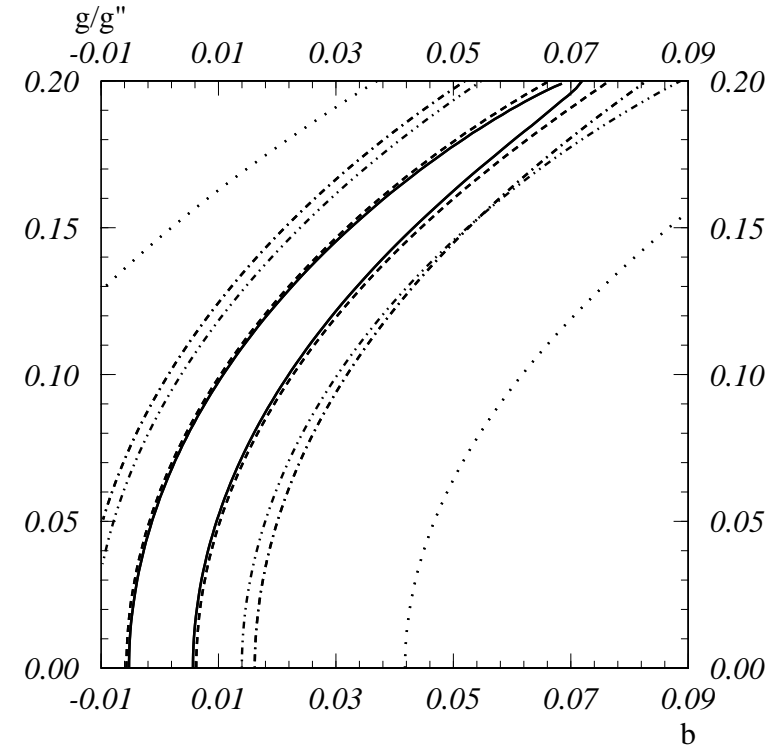

Figure 5: Same of Figure 1 for the parameter space $\left(b, g / g^{\prime \prime}\right)$ in the case of model 3 (BESS model).

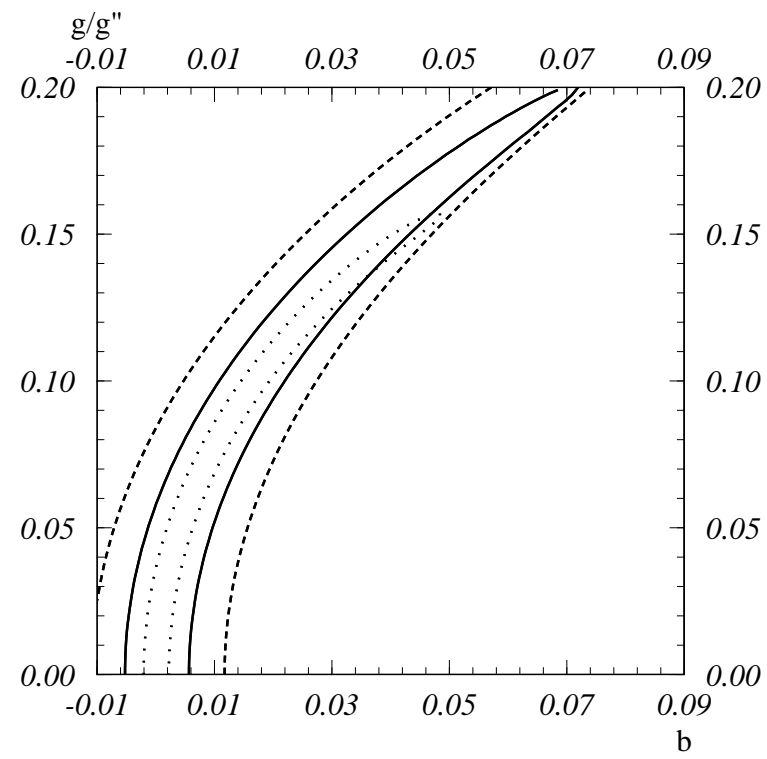

Figure 6: Same of Figure 2 for the parameter space $\left(b, g / g^{\prime \prime}\right)$ in the case of model 3 (BESS model). 


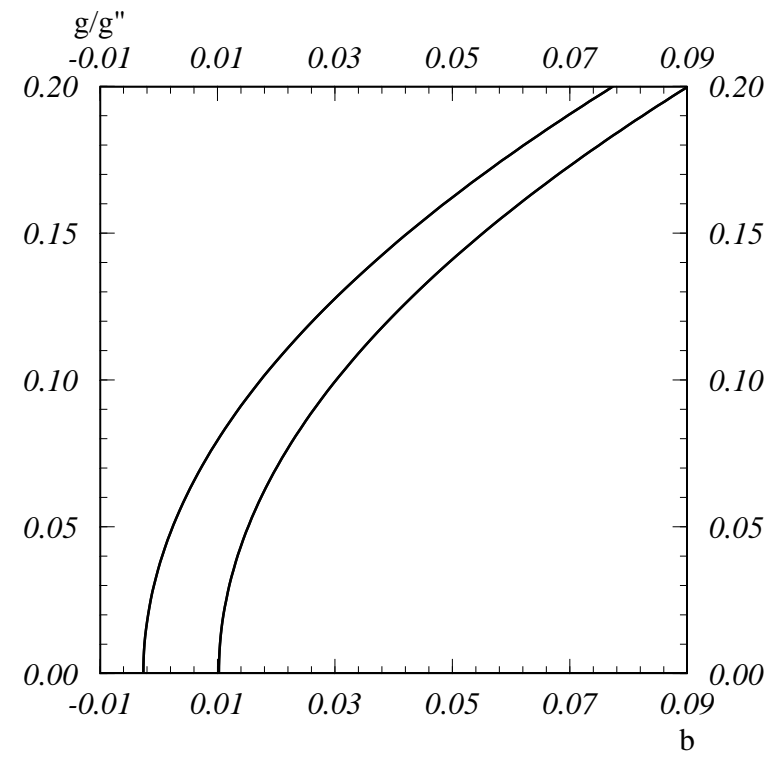

Figure 7: $90 \%$ C.L. bounds on the parameter space $\left(b, g / g^{\prime \prime}\right)$ in the case of model 3 (BESS model), as obtained from LEP1 experiments.

\section{MODEL 3}

Let us now consider the so called BESS model (see ref. [11). This is an effective model in which the existence of a new triplet of vector fields $\vec{V}_{\mu}$ is assumed. These are the gauge fields associated to a spontaneously broken local symmetry $S U(2)_{V}$. The new vector particles mix with $W$ and $Z$. As a consequence at energies well below their mass (assumed to be around the $T e V$ scale), effective anomalous fermionic and trilinear couplings are generated (see ref. [12]). The parameters characterizing this model are the gauge coupling of the $\vec{V}_{\mu}, g^{\prime \prime}$, and their direct coupling to the fermions, $b$. Only $\epsilon_{3}$ and $\delta_{z}$ are different from zero and their are given by

$$
\begin{gathered}
\epsilon_{3}=-\frac{b}{2}+\left(\frac{g}{g^{\prime \prime}}\right)^{2} \\
\delta_{z}=\frac{c_{\theta}}{2 s_{\theta} c_{2 \theta}}\left(b-\frac{1}{c_{\theta}^{2}}\left(\frac{g}{g^{\prime \prime}}\right)^{2}\right)
\end{gathered}
$$

In Figs. 5 and 6 we give the $90 \%$ C.L. bounds in the parameter space $\left(b, g / g^{\prime \prime}\right)$ again at fixed energy and different observables and at various energies respectively. Also in this case $\sigma_{L L}$ results to be the most restrictive observable. The BESS model is a generalization of a non-linear $\sigma$-model and it is not renormalizable. However at 1-loop level it is equivalent to a heavy Higgs model with the Higgs mass playing the role of a cut-off. Assuming the 


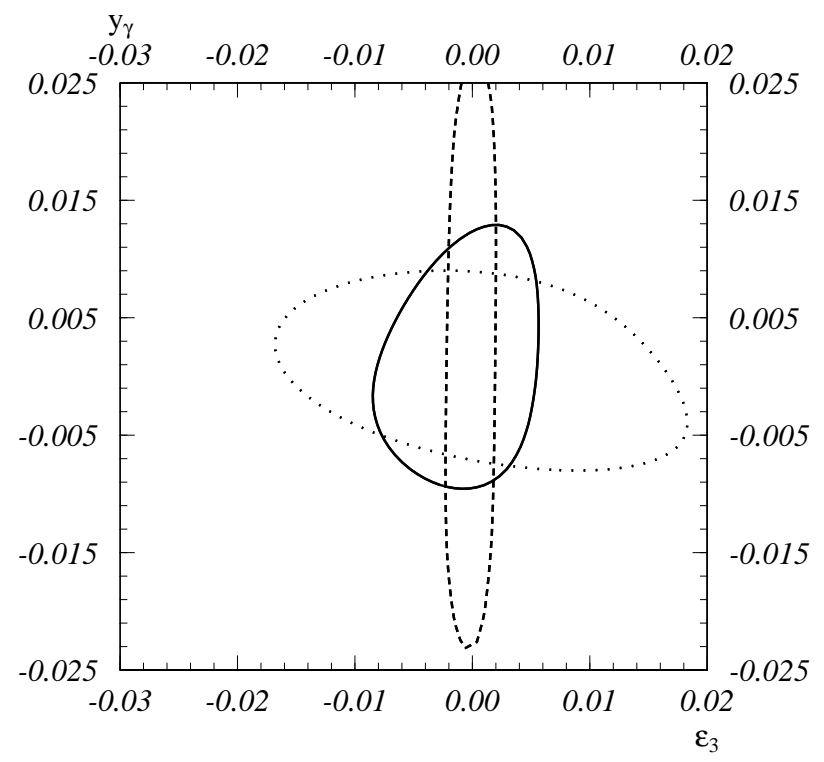

Figure 8: $90 \%$ C.L. bounds on the parameter space $\left(\epsilon_{3}, y_{\gamma}\right)$ in the case of model 1 . The bounds are obtained from $\sigma_{\text {comb }}$ at $E=500 \mathrm{GeV}$ and $L=20 \mathrm{fb}^{-1}$, and different degrees of polarization: the dashed line corresponds to $P_{e}=0.9$, the continuous one to $P_{e}=0$, and the dotted one to $P_{e}=-0.9$.

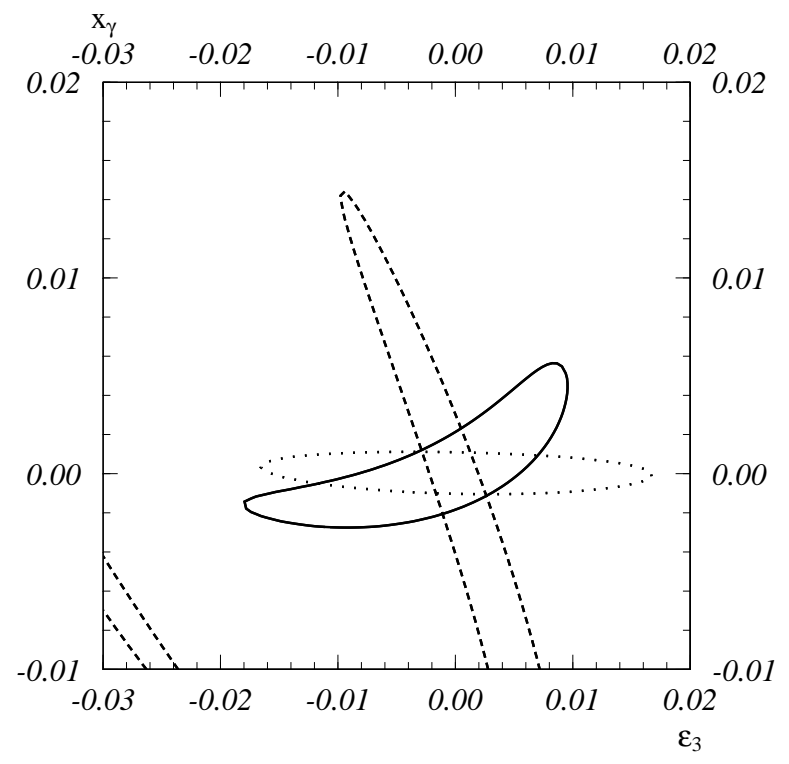

Figure 9: Same of Figure 8 for the parameter space $\left(\epsilon_{3}, x_{\gamma}\right)$ in the case of model 2. 


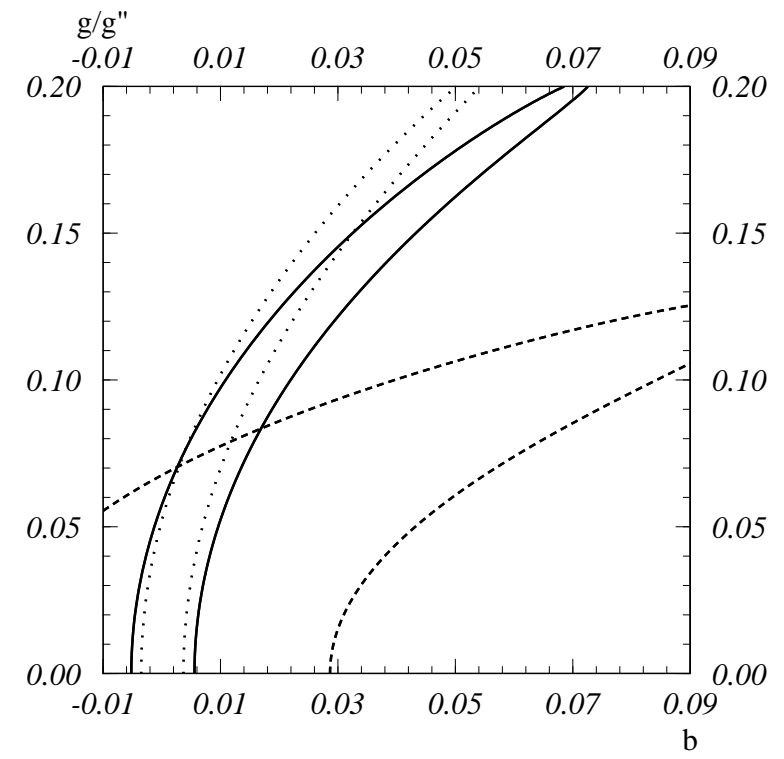

Figure 10: Same of Figure 8 for the parameter space $\left(b, g / g^{\prime \prime}\right)$ in the case of model 3 (BESS model).

cut-off of the order of $1 \mathrm{TeV}$ we can evaluate $\epsilon_{3}^{\mathrm{rad}}$ with a Higgs mass equal to $1 \mathrm{TeV}$. In Fig. 7 we give the bounds obtained in this way on the parameter space arising from LEP1 experiments. The comparison with Fig. 6 shows the need of reaching an energy of at least $500 \mathrm{GeV}$ in order to get a real improvement.

Finally we have considered the possibility of polarized electron beams. In Figs. 8, 9 and 10 we show, for the previous models, the $90 \%$ C.L. bounds from $\sigma_{\text {comb }}$ in the cases of $P_{e}=$ $0, \pm 0.9$. We see that the general effect of the polarization is to select different combinations of the anomalous parameters producing, as a consequence, a rotation of the allowed region. In this way, combining polarized and non polarized differential cross-sections one is able to get significantly more restrictive bounds on the anomalous parameters.

\section{Conclusions}

The main point of this paper is the inclusion of non-standard fermionic couplings on the same footing of the anomalous gauge ones in the phenomenological analysis of the $e^{+} e^{-} \rightarrow W^{+} W^{-}$process for different options of the next linear colliders. This is justified by the fact that new physics will change generally both types of couplings, and also from the LEP1 results, that strongly encourage the hypothesis that, if they are there, then they should be of the same order of magnitude. The analysis shows also that the these two different types of non-standard couplings are strongly correlated. 
We have also studied the high-energy behaviour of the differential cross-sections showing that there is a quadratic contribution from the anomalous couplings which dominates the limit. Therefore a linear approximation in the anomalous terms is not justified, and, in fact, all our numerical analysis is done by using the exact expressions.

In summary we have seen that the NLC (specially in the higher energy option considered here) can give very stringent bounds on the anomalous parameters also improving our knowledge of the fermionic couplings. Furthermore, if polarized electron beams will be available, the bounds will increase dramatically.

\section{Appendix A}

We give here the list of the coefficients $F^{(\alpha)}$ appearing in the high-energy expansion of the helicity amplitudes for $\alpha=1,1 / 2,0,-1 / 2,-1$. We give separately the anomalous contributions and the SM ones.

\section{Longitudinal-Longitudinal Case}

$$
\begin{aligned}
& \delta F_{0,0}^{1 / 2,-1 / 2(1)}=-\frac{e^{2}}{4} \sin \phi \frac{1}{M_{W}^{2}}\left(\cot \theta\left(\delta a_{e}^{Z}-\delta b_{e}^{Z}\right)+\tan \theta\left(\delta_{z}+x_{z}\right)-x_{\gamma}\right) \\
& F_{0,0}^{1 / 2,-1 / 2(0)}(S M)=-\frac{e^{2}}{4} \sin \phi \frac{1}{c_{\theta}^{2}} \\
& \delta F_{0,0}^{1 / 2,-1 / 2(0)}=-\frac{e^{2}}{4} \sin \phi\left(-\frac{2}{c_{\theta}^{2}} \frac{\delta M_{W}}{M_{W}}+\frac{1}{s_{\theta} c_{\theta}}\left(\delta a_{e}^{Z}-\delta b_{e}^{Z}\right)\right. \\
& \left.+\delta_{Z} \frac{\tan \theta}{c_{\theta}^{2}}-x_{z} \tan \theta \frac{1-2 s_{\theta}^{2}}{c_{\theta}^{2}}+2 x_{\gamma}\right) \\
& F_{0,0}^{1 / 2,-1 / 2(-1)}(S M)=-\frac{e^{2}}{4} \sin \phi \frac{M_{W}^{2}}{c_{\theta}^{4}} \\
& \delta F_{0,0}^{-1 / 2,1 / 2(1)}=\frac{e^{2}}{4} \sin \phi \frac{1}{M_{W}^{2}}\left(-\frac{2 \sqrt{2} \delta a_{W}}{s_{\theta}}-\cot \theta\left(\delta a_{e}^{Z}+\delta b_{e}^{Z}\right)\right. \\
& \left.+\cot 2 \theta\left(\delta_{z}+x_{z}\right)+x_{\gamma}\right) \\
& F_{0,0}^{-1 / 2,1 / 2(0)}(S M)=-\frac{e^{2}}{2} \sin \phi \frac{1}{s_{2 \theta}^{2}} \\
& \delta F_{0,0}^{-1 / 2,1 / 2(0)}=\frac{e^{2}}{4} \sin \phi\left(-4 \frac{\cos 2 \theta}{\sin ^{2} 2 \theta} \frac{\delta M_{W}}{M_{W}}-\frac{1}{s_{\theta} c_{\theta}}\left(\delta a_{e}^{Z}+\delta b_{e}^{Z}\right)\right.
\end{aligned}
$$




$$
\begin{array}{r}
\left.-\frac{4 \sqrt{2}}{s_{\theta}} \delta a_{W}+\frac{\cot 2 \theta}{c_{\theta}^{2}}\left(\delta_{z}-x_{z} c_{2 \theta}\right)-2 x_{\gamma}\right) \\
F_{0,0}^{-1 / 2,1 / 2(-1)}(S M)=-\frac{e^{2}}{4} \sin \phi \frac{M_{W}^{2}}{s_{\theta}^{2}}\left(-\frac{c_{2 \theta}}{2 c_{\theta}^{4}}+2 \frac{1-3 \cos \phi}{1-\cos \phi}\right)
\end{array}
$$

\section{Longitudinal-Transverse Case}

$$
\begin{aligned}
& \delta F_{1,0}^{1 / 2,-1 / 2(1 / 2)}=\frac{e^{2}}{4 \sqrt{2}}(\cos \phi+1) \frac{1}{M_{W}}\left(2 \cot \theta\left(\delta a_{e}^{Z}-\delta b_{e}^{Z}\right)\right. \\
& \left.-\left(x_{\gamma}+y_{\gamma}\right)+\tan \theta\left(x_{z}+y_{z}+2 \delta_{z}\right)\right) \\
& F_{1,0}^{1 / 2,-1 / 2(-1 / 2)}(S M)=\frac{e^{2}}{2 \sqrt{2}}(\cos \phi+1) \frac{M_{W}}{c_{\theta}^{2}} \\
& \delta F_{1,0}^{-1 / 2,1 / 2(1 / 2)}=\frac{e^{2}}{4 \sqrt{2}}(\cos \phi-1) \frac{1}{M_{W}}\left(-\frac{4 \sqrt{2}}{s_{\theta}} \delta a_{W}-2 \cot \theta\left(\delta a_{e}^{Z}+\delta b_{e}^{Z}\right)\right. \\
& \left.+\left(x_{\gamma}+y_{\gamma}\right)+\cot 2 \theta\left(x_{z}+y_{z}+2 \delta_{z}\right)\right) \\
& F_{1,0}^{-1 / 2,1 / 2(-1 / 2)}(S M)=-\frac{e^{2}}{\sqrt{2}}(\cos \phi-1)\left(\cot 2 \theta+\frac{1}{s_{\theta}^{2}} \frac{\cos \phi}{1-\cos \phi}\right) M_{W} \\
& \delta F_{-1,0}^{1 / 2,-1 / 2(1 / 2)}=\frac{e^{2}}{4 \sqrt{2}}(\cos \phi-1) \frac{1}{M_{W}}\left(-2 \cot \theta\left(\delta a_{e}^{Z}-\delta b_{e}^{Z}\right)\right. \\
& \left.+\left(x_{\gamma}+y_{\gamma}\right)-\tan \theta\left(x_{z}+y_{z}+2 \delta_{z}\right)\right) \\
& F_{-1,0}^{1 / 2,-1 / 2(-1 / 2)}(S M)=\frac{e^{2}}{2 \sqrt{2}}(-\cos \phi+1) \frac{M_{W}}{c_{\theta}^{2}} \\
& \delta F_{-1,0}^{-1 / 2,1 / 2(1 / 2)}=\frac{e^{2}}{4 \sqrt{2}}(\cos \phi+1) \frac{1}{M_{W}}\left(\frac{4 \sqrt{2}}{s_{\theta}} \delta a_{W}+2 \cot \theta\left(\delta a_{e}^{Z}+\delta b_{e}^{Z}\right)\right. \\
& \left.-\left(x_{\gamma}+y_{\gamma}\right)-\cot 2 \theta\left(x_{z}+y_{z}+2 \delta_{z}\right)\right) \\
& F_{-1,0}^{-1 / 2,1 / 2(-1 / 2)}(S M)=-\frac{e^{2}}{4 \sqrt{2}}(\cos \phi+1) M_{W} \frac{1+2 c_{\theta}^{2}}{s_{\theta}^{2} c_{\theta}^{2}}
\end{aligned}
$$

\section{Transverse -Transverse Case}

$$
\begin{aligned}
& \delta F_{1,1}^{1 / 2,-1 / 2(1)}=\frac{e^{2}}{4} \sin \phi \frac{1}{M_{W}^{2}}\left(y_{\gamma}-y_{z} \tan \theta\right) \\
& F_{1,1}^{1 / 2,-1 / 2(0)}(S M)=0
\end{aligned}
$$




$$
\begin{aligned}
& \delta F_{1,1}^{1 / 2,-1 / 2(0)}=-\frac{e^{2}}{4} \sin \phi\left(2 \cot \theta\left(\delta a_{e}^{Z}-\delta b_{e}^{Z}\right)\right. \\
& \left.+2 y_{\gamma}+2 \delta_{z} \tan \theta-y_{z} \tan \theta \frac{c_{2 \theta}}{c_{\theta}^{2}}\right) \\
& F_{1,1}^{1 / 2,-1 / 2(-1)}(S M)=-\frac{e^{2}}{2} \sin \phi \frac{M_{W}^{2}}{c_{\theta}^{2}} \\
& \delta F_{1,1}^{-1 / 2,1 / 2(1)}=\frac{e^{2}}{4} \sin \phi \frac{1}{M_{W}^{2}}\left(y_{\gamma}+y_{z} \cot 2 \theta\right) \\
& F_{1,1}^{-1 / 2,1 / 2(0)}(S M)=0 \\
& \delta F_{1,1}^{-1 / 2,1 / 2(0)}=\frac{e^{2}}{4} \sin \phi\left(-\frac{4 \sqrt{2}}{s_{\theta}} \delta a_{W}-2 \cot \theta\left(\delta a_{e}^{Z}+\delta b_{e}^{Z}\right)\right. \\
& \left.+2 \delta_{z} \cot 2 \theta-2 y_{\gamma}-y_{z} \cot 2 \theta \frac{c_{2 \theta}}{c_{\theta}^{2}}\right) \\
& F_{1,1}^{-1 / 2,1 / 2(-1)}(S M)=\frac{e^{2}}{4} \sin \phi M_{W}^{2}\left(-\frac{1}{s_{\theta}^{2} c_{\theta}^{2}}+\frac{1}{s_{\theta}^{2}} \frac{2 \cos \phi}{1-\cos \phi}\right) \\
& \delta F_{1,-1}^{-1 / 2,1 / 2(1)}=0 \\
& F_{1,-1}^{-1 / 2,1 / 2(0)}(S M)=\frac{e^{2}}{4} \sin \phi \frac{1}{s_{\theta}^{2}} \\
& \delta F_{1,-1}^{-1 / 2,1 / 2(0)}=e^{2} \sin \phi \frac{\sqrt{2}}{s_{\theta}} \delta a_{W} \\
& F_{-1,1}^{-1 / 2,1 / 2(-1)}(S M)=-\frac{e^{2}}{2} \sin \phi \frac{M_{W}^{2}}{s_{\theta}^{2}} \frac{1+\cos \phi}{1-\cos \phi} \\
& F_{1,-1}^{-1 / 2,1 / 2(-1)}(S M)=\frac{e^{2}}{2} \sin \phi \frac{M_{W}^{2}}{s_{\theta}^{2}} \\
& \delta F_{-1,1}^{-1 / 2,1 / 2(1)}=0 \\
& F_{-1,1}^{-1 / 2,1 / 2(0)}(S M)=-\frac{e^{2}}{4} \sin \phi \frac{1}{s_{\theta}^{2}} \frac{1+\cos \phi}{1-\cos \phi} \\
& \delta F_{-1,1}^{-1 / 2,1 / 2(0)}=-e^{2} \sin \phi \frac{\sqrt{2}}{1+\cos \phi} \delta a_{W} \\
& \left.\begin{array}{l}
s_{\theta} \\
1-\cos \phi
\end{array}\right)
\end{aligned}
$$

\section{ACKNOWLEDGMENTS}

This work is part of the EEC Project "Tests of electroweak symmetry breaking and future European colliders", No. CHRXCT94/0579.

\section{References}


[1] H. Murayama and M.E. Peskin, SLAC-PUB-7149, hep-ex/9606003, June 1996, to appear in Annual Reviews of Nuclear and Particle Science.

[2] K. Hagiwara, R. Peccei, D. Zeppenfeld and K. Hikasa, Nucl. Phys. B282 (1987) 253. For a recent review see: H. Aihara et al. MAD/PH/871, hep-ph/9503425, Summary of the Working Group on Anomalous Gauge Interactions of the DPF Long-Range Planning Study, to be published in Electroweak Symmetry Breaking and Beyond the Standard Model, eds. T. Barklow, S. Dawson, H. Haber and J. Siegrist.

[3] M. Bilenky, J.L. Kneur, F.M. Renard and D. Schildknecht, Nucl. Phys. B409 (1993) 22; A. Blondel et al., Proceedings of the ECFA Workshop on LEP 200, Aachen, 1986, eds. A. Böhm, and W. Hoogland, CERN Report 87-08 (1987), Vol. I, p. 120; M. Frank, P. Mättig, R. Settles and W. Zeuner, Proceedings of the Workshop $e^{+} e^{-}$ Collisions at 500 GeV: the Physics Potential, ed. P.M. Zerwas (1992), DESY 92123B, p. 223.

[4] V.V. Andreev, A.A. Pankov and N. Paver, Phys. Rev. D53 (1995) 2390, hep$\mathrm{ph} / 9511300$.

[5] See: K. Hagiwara et. al. in [2], and M. Bilenky et al. in [3].

[6] See: K. Hagiwara et. al. in [2]; G. Gounaris et al., CERN Report on Physics at LEP2, CERN 96-01 (1996), eds. G. Altarelli, T. Sjöstrand and F. Zwirner, Vol. I, p. 525.

[7] See: M. Frank et al. in [3]; R.W. Forty, J.B. Hansen, J.D. Hansen and R. Settles, Collisions at $500 \mathrm{GeV}$ : the Physics Potential, ed. P.M. Zerwas (1993), DESY 93-123c, p. 235; R. Settles, ibidem, p. 591; H. Anlauf, A. Himmler, P. Manakos, T. Mannel and H. Dahmen, Proceedings of the Workshop Physics and Experiments with Linear $e^{+} e^{-}$Colliders, Waikoloa, Hawaii, p. 708, eds. F.A. Harris, S.L. Holsen, S. Pakvasa and X. Tata, World Scientific, Singapore (1993).

[8] G. Altarelli and R. Barbieri, Phys. Lett. B253 (1991) 161; G. Altarelli, R. Barbieri and S. Jadach, Nucl. Phys. B369 (1992) 3.

[9] F. Caravaglios, talk at the EPS Conference on Particle Physics, Bruxelles, July 1995.

[10] See M. Bilenky et al. in [3].

[11] R.Casalbuoni, S.De Curtis, D.Dominici and R.Gatto, Phys. Lett. B155 (1985) 95; and Nucl. Phys. B282 (1987) 235.

[12] L. Anichini, R. Casalbuoni and S. De Curtis, Phys. Lett. B348 (1995) 521, hepph/9410377. 